\title{
Translation of Inhaled Drug Optimization Strategies into Clinical Pharmacokinetics and Pharmacodynamics Using GSK2292767A, a Novel Inhaled Phosphoinositide 3-Kinase $\delta$ Inhibitor $\$$
}

\author{
Malcolm Begg, ${ }^{1}$ Chris D. Edwards, ${ }^{1}$ J. Nicole Hamblin, Eleni Pefani, Robert Wilson, \\ Jane Gilbert, Giovanni Vitulli, David Mallett, Josie Morrell, Martin I. Hingle, Sorif Uddin, \\ Filzah Ehtesham, Miriam Marotti, Andrew Harrell, Carla F. Newman, Disala Fernando, \\ Jonathan Clark, Anthony Cahn, and Edith M. Hessel
}

Refractory Respiratory Inflammation Discovery Performance Unit, GlaxoSmithKline, Stevenage, United Kingdom (M.B., C.D.E., J.N.H., E.M.H.); Clinical Pharmacology \& Model Stimulation, (E.P., R.W.), Drug Metabolism \& Pharmacokinetics, (G.V., D.M., J.M.), Refractory Respiratory Inflammation DPU, (S.U.), In Vitro In Vivo Translation, (C.F.N.), Discovery Medicine, (A.C) GlaxoSmithKline, Stevenage, United Kingdom; Global Clinical Science \& Delivery, GlaxoSmithKline, Stockley Park, Uxbridge, United Kingdom (M.M., J.G.); In Vitro In Vivo Translation, (A.H.), Drug Product Design \& Development, (M.I.H.), GlaxoSmithKline, Ware, United Kingdom (M.I.H.); Department of Biological Chemistry, Babraham Institute, Cambridge, United Kingdom (J.C.); and Clinical Unit Cambridge, Addenbrookes Hospital, Cambridge, United Kingdom (F.E., D.F.)

Received February 14, 2019; accepted March 27, 2019

\section{ABSTRACT}

This study describes the pharmacokinetic $(\mathrm{PK})$ and pharmacodynamic (PD) profile of $N$-(5-(4-(5-(((2R,6S)-2,6-dimethylmorpholino)methyl)oxazol-2-yl)-1H-indazol-6-yl)-2-methoxypyridin-3-yl)methanesulfonamide (GSK2292767A), a novel low-solubility inhaled phosphoinositide 3-kinase delta $(\mathrm{PI} 3 \mathrm{~K} \delta)$ inhibitor developed as an alternative to $2-(6-(1 \mathrm{H}$-indol-4-yl)-1H-indazol4-yl)-5-((4-isopropylpiperazin-1-yl)methyl)oxazole (nemiralisib), which is a highly soluble inhaled inhibitor of PI3K $\delta$ with a lung profile consistent with once-daily dosing. GSK2292767A has a similar in vitro cellular profile to nemiralisib and reduces eosinophilia in a murine PD model by $63 \%(n=5, P<0.05)$. To explore whether a low-soluble compound results in effective $\mathrm{PI} 3 \mathrm{~K} \delta$ inhibition in humans, a first time in human study was conducted with GSK2292767A in healthy volunteers who smoke. GSK2292767A was generally well tolerated, with headache being the most common reported adverse event. PD changes in induced

This work was funded by GlaxoSmithKline.

M.B., E.P., R.W., E.M.H., M.I.H., C.E., G.V., C.F.N., D.M., M.M., A.W.H., F.E., A.C., and J.M. are employees of GlaxoSmithKline (GSK) and hold shares in the company. D.F. owns GSK stocks and shares. J.G. is an employee of Parasol and contracted to work solely for GSK. J.N.H. was an employee of GSK at the time the work was carried out and holds shares in the company. J.C. and S.U. have no conflicts of interest to declare.

Anonymized individual participant data and documents from study $202062 \mathrm{can}$ be requested for further research from www.clinicalstudydatarequest.com.

${ }^{1}$ M.B. and C.D.E. contributed equally to this work

https://doi.org/10.1124/jpet.119.257311.

S This article has supplemental material available at jpet.aspetjournals.org. sputum were measured in combination with drug concentrations in plasma from single $(0.05-2 \mathrm{mg}, n=37)$, and 14-day repeat (2 mg, $n=12$ ) doses of GSK2292767A. Trough bronchoalveolar lavage $(B A L)$ for $P K$ was taken after 14 days of repeat dosing. GSK2292767A displayed a linear increase in plasma exposure with dose, with marginal accumulation after 14 days. Induced sputum showed a $27 \%$ (90\% confidence interval $15 \%, 37 \%)$ reduction in phosphatidylinositol-trisphosphate (the product of phosphoinositide 3-kinase activation) 3 hours after a single dose. Reduction was not maintained 24 hours after single or repeat dosing. BAL analysis confirmed the presence of GSK2292767A in lung at 24 hours, consistent with the preclinical lung retention profile. Despite good lung retention, target engagement was only present at 3 hours. This exposure-response disconnect is an important observation for future inhaled drug design strategies considering low solubility to drive lung retention.

ABBREVIATIONS: AE, adverse event; AUC, area under the plasma concentration-time curve; BAL, bronchoalveolar lavage; Cl, confidence interval; ELF, epithelial lining fluid; GSK2292767A, N-(5-(4-(5-((2R,6S)-2,6-dimethylmorpholino)methyl)oxazol-2-yl)-1H-indazol-6-yl)-2-methoxypyridin-3-yl) methanesulfonamide; HDM, house dust mite; LC-MS/MS, liquid chromatography-tandem mass spectrometry; 2- $(6-(1 \mathrm{H}$-indol-4-yl)-1H-indazol-4yl)-5-((4-isopropylpiperazin-1-yl)methyl)oxazole; PD, pharmacodynamic; PIP2, phosphatidylinositol-biphosphate; PIP3, phosphatidylinositoltrisphosphate; PI3K $\delta$, phosphoinositide 3-kinase delta; PK, pharmacokinetic; $t_{\max }$, time to maximum observed plasma concentration. 
facilitating drug delivery to its biologic target. This can be achieved either by lung tissue binding or intracellular uptake with highly soluble molecules, or alternatively by slow in situ dissolution with poorly soluble molecules. We have recently described the pharmacokinetic (PK) and pharmacodynamic (PD) profile of 2-(6-(1H-indol-4-yl)-1H-indazol-4-yl)-5-((4-isopropylpiperazin-1-yl)methyl)oxazole (nemiralisib), an inhaled inhibitor of phosphoinositide 3-kinase delta $(\mathrm{PI} 3 \mathrm{~K} \delta$ ), which was designed as a basic, soluble molecule with moderate lipophilicity. This physicochemical profile resulted in a oncedaily dose regimen (Begg et al., 2019). Nemiralisib is currently being developed for respiratory diseases as an immune modulator with anti-inflammatory properties (Stark et al., 2015; Cahn et al., 2017; Khindri et al., 2018).

Here, we have adopted an alternative approach for driving lung retention through reduced molecule solubility, resulting in the novel structurally distinct PI3K $\delta$ inhibitor $N$-(5-(4-(5$(((2 R, 6 S)-2,6$-dimethylmorpholino)methyl)oxazol-2-yl)- $1 H$ indazol-6-yl)-2-methoxypyridin-3-yl)methanesulfonamide (GSK2292767A). This strategy has been previously employed by others (Hochhaus et al., 1995, 1997), and relies on slow dissolution of inhaled particles to drive lung retention. In contrast, the strategy used for nemiralisib introduced basicity, which either maintained levels of dissolved compound in the lung through trapping in acidic organelles such as lysosomes (Perry et al., 2017), or delivered lung retention through high lung tissue binding. Duration of action in the lung relies on slow release, either from depots of particulate material for low-solubility molecules or dissolved material trapped in lysosomes or bound to tissue with soluble molecules.

In this study, we report a detailed profile of the PK and PD relationship of the low-solubility compound GSK2292767A and set out to explore whether the lung retention strategy was successful by investigating the corresponding PD response in vitro, in vivo, and in a clinical trial. GSK2292767A and nemiralisib have broadly similar profiles in terms of in vitro potency, selectivity, and in vivo efficacy, and were shown to have suitable PK properties for inhaled delivery including low oral bioavailability (Down et al., 2015). Our prior knowledge of the profile of nemiralisib and the current detailed research reported here for GSK2292767A, provides a unique opportunity to compare and contrast two lung retention strategies and their resulting inhaled clinical profiles. This paper is also the first full disclosure of the clinical safety profile, tolerability, and target engagement profile of GSK2292767A.

\section{Materials and Methods}

\section{Preclinical Methods}

Compound Solubility and Dissolution Profiles. Saturation solubility studies were performed in simulated lung fluid, a phosphate-buffered saline containing lecithin and bovine serum albumin at naturally occurring lung lining fluid $\mathrm{pH}$. The amount of three different salt forms of GSK2292767 (version A = free base, version $\mathrm{C}=$ sodium salt, and version $\mathrm{K}=$ cinnamate salt) and nemiralisib added into the media was excessive, wetted by sonication for 5 minutes, and all suspensions were kept shaking at $100 \mathrm{rpm}$ on an orbital shaker $\left(37^{\circ} \mathrm{C}\right)$ for 24 hours. The suspensions were centrifuged at $14,000 \mathrm{rpm}$ for 10 minutes and filtered through a $0.22 \mu \mathrm{m}$ polyvinylidene difluoride filter.

For dissolution testing, an abbreviated Next Generation Impactor (NGI) was used to collect dose. The fine particle fraction of micronized
GSK2292767 salt forms was collected above next-generation impactor stage 3 (Riley et al., 2012) onto a filtrete for aerosol dissolution. Micronized active pharmaceutical ingredients were filled into gelatin capsules and dispersed with a HandiHaler (Boehringer Ingelheim) into the next-generation impactor at a $60 \mathrm{l} / \mathrm{min}$ airflow rate with 4-second exposure. A previously reported USP intravenous apparatus was used to perform aerosol dissolution work (Davies and Feddah, 2003) with simulated lung fluid as the dissolution media. After dose collection, the drug-loaded filtrete was transferred to the dissolution cell, placed in the dissolution oven, and allowed to equilibrate. Dissolution testing was performed at $37^{\circ} \mathrm{C}$ with a simulated lung fluid flow rate of $1 \mathrm{ml} / \mathrm{min}$. At the end of the experimental procedure all parts of the dissolution cell were washed with a defined amount of solvent [acetonitrile:water (50:50)] to determine total recovery.

All saturation solubility and dissolution samples were quantified via high-performance liquid chromatography. High-performance liquid chromatography analysis was performed using an Agilent 1100 system with UV-visible detection.

Cell Permeability. GSK2292767A and nemiralisib (GSK2269557B, $\mathrm{HCl}$ salt form) were applied to the apical side of a confluent monolayer of Madin-Darby canine kidney cells transfected with the efflux transporter MDR1 (MDR1/Madin-Darby canine kidney II cells), in duplicate at a concentration of $3 \mu \mathrm{M}$ Dulbecco's Modified Eagle's Medium (DMEM) buffer at $\mathrm{pH} 7.4$ as both donor and receiver solutions. Incubations were carried out in an atmosphere of $5 \% \mathrm{CO}_{2}$ with relative humidity of $95 \%$ at $37^{\circ} \mathrm{C}$ for 90 minutes. The permeability was measured from the apical to the basolateral side of the cell membrane in the presence of the MDR1 inhibitor GF120918A，(N-(4-(2-(6,7-dimethoxy-3,4-dihydroisoquinolin-2(1H)-yl)ethyl) phenyl)-5-methoxy-9-oxo-9,10-dihydroacridine-4-carboxamide). Quantification of parent compound in transport media was determined following analysis by liquid chromatography-tandem mass spectrometry (LCMS/MS).

Lung Tissue Binding. All animal studies were ethically reviewed and carried out in accordance with the Animals (Scientific Procedures) Act 1986 and the GlaxoSmithKline policy on the care, welfare, and treatment of animals.

The binding of GSK2292767A and nemiralisib (GSK2269557B) was measured in male $\mathrm{CD}$ rat lungs and in female BALB/c mouse lungs and blood in vitro, using rat and mouse lung homogenate and mouse whole blood, by rapid equilibrium dialysis at the nominal concentration of $1 \mu \mathrm{g} / \mathrm{ml}$. The test compound was spiked into lung homogenate diluted 1:9, with dialysis buffer (PBS $-100 \mathrm{mM}$ sodium phosphate + $150 \mathrm{mM}$ sodium chloride, $\mathrm{pH}$ 6.9-7.2) or whole blood (diluted 1:1 with dialysis buffer) and incubated for 4 hours at $37^{\circ} \mathrm{C}$ in five replicates using rapid equilibrium dialysis plates (Thermo Scientific, Rockford, IL). The matrix was on one side on the dialysis membrane with the dialysis buffer on the other. All buffer, lung homogenate, and blood samples $(50 \mu \mathrm{l})$ were matrix matched by diluting 2 -fold with control lung homogenate, blood, or buffer, and the resultant samples were extracted by protein precipitation. The lung homogenate and blood binding of GSK2292767A was determined by peak area ratio following LC-MS/MS analysis. The lung tissue binding was then determined using an in-house validated method previously described for brain homogenates (Kalvass and Maurer, 2002).

Lung and Blood PK Profiles Following Intranasal Delivery to the Mouse. Eighteen female BALB/c mice were placed under anesthesia using isoflurane to facilitate intranasal administration of GSK2292767A or nemiralisib (GSK2269557B) at $1 \mathrm{mg}$ base equivalent $/ \mathrm{kg}$, as a $50 \mu \mathrm{l}$ volume of a $0.4 \mathrm{mg} / \mathrm{ml}$ suspension formulated in $0.2 \%$ Tween 80 , which was in saline adjusted to $\mathrm{pH} 4.5$ with $1 \mathrm{M} \mathrm{HCl}(\sim 7 \mu \mathrm{l})$. Terminal lung and blood samples were collected at 10 and 30 minutes, and 1, 3, 5, and 8 hours after dosing ( $n=3$ per time point). Concentration-time profiles were used to determine the PK parameters in both blood and lung via noncompartmental analysis. Unbound concentrations were derived by correcting for in vitro binding data generated in mouse whole blood and mouse lung homogenate.

Mouse lungs along with $1 \mathrm{ml}$ water were homogenized with ceramic beads using a Precellys 24 homogenizer (Bertin Technologies) for two 
20 second cycles at $6500 \mathrm{rpm}$ separated by a 10 second pause, with a further $1 \mathrm{ml}$ water then added. Lung homogenates and blood samples underwent protein precipitation with $100 \%$ acetonitrile containing internal standard followed by centrifugation. Aliquots of the resultant supernatants were dried under heated nitrogen $\left(37^{\circ} \mathrm{C}\right)$, and then reconstituted in $200 \mu \mathrm{l}$ of acetonitrile:water (10:90 v/v) before being analyzed by LC-MS/MS.

Lung Retention Profiles Following Inhaled Delivery to the Rat. The PK in lung was measured for the free base, sodium salt, and cinnamate salt forms of GSK2292767 after a single administration of each version as a micronized dry powder, blended to $15 \%$ active pharmaceutical ingredients with lactose, to the male Wistar Han rat at a nominal dose of $0.6 \mathrm{mg} / \mathrm{kg}$. For each version, the drug was delivered via an aerosolized dust generation inhalation tower connected to a small cup Wrights Dust Feeder II aerosol generator, with a target inhalation period of 20 minutes. Twenty-one rats were exposed for each study, with termination ( $n=3$ rats/time point) scheduled at the following time points measured from the end of the aerosol delivery: -10 (half-way through drug administration), and 0 minutes immediately after dosing and 2, 4, 8, 12, and 24 hours. Aqueous tissue homogenates were obtained from all lung samples, using a Precellys 24 device (two 20 second cycles at $6500 \mathrm{rpm}$ separated by a 10 second pause).

The PK in lung was measured for nemiralisib (GSK2269557H, hemisuccinate salt), after a single intratracheal administration by Penn-Century insufflation as a micronized dry powder, blended to $15 \%$ active pharmaceutical ingredients with lactose, to the male Brown Norway rat at a nominal dose of $0.67 \mathrm{mg} / \mathrm{kg}$. Nine rats were exposed in total, with termination and collection of lungs ( $n=3 \mathrm{rats} /$ time point) scheduled at the following times: 0 minutes immediately after dosing 7 and 24 hours. Aqueous tissue homogenates were obtained from all lung samples, using a Precellys device as described previously. Levels of GSK2292767A and nemiralisib were measured in lung homogenate samples prepared by protein precipitation followed by LC-MS/MS analysis.

House Dust Mite Rechallenge Model of Pulmonary Inflammation. Female BALB/c mice were sensitized to house dust mite [(HDM), Dermatophagoides pteronyssinus; Greer Laboratories, Lenoir, NC] by intranasal dosing, once a day for 5 days a week over a 21-day period with $25 \mu \mathrm{g}$ HDM extract (Greer Laboratories). Pulmonary inflammation was then allowed to resolve until day 33. Inflammation was then reinitiated by an intranasal rechallenge of $\mathrm{HDM}$ using $100 \mu \mathrm{g}$ of HDM. GSK2292767A and nemiralisib (GSK2269557H) were administered as repeat daily doses of inhaled dry powder lactose blends for 7 days prior to rechallenge and their effect on inflammatory eosinophils was ascertained by flow cytometry on bronchoalveolar lavage (BAL) samples.

\section{Clinical Trial Methods}

First Time in Human Subject Population. This first time in human study (GSK Protocol 202062, NCT03045887) was conducted at the Clinical Unit Cambridge (Cambridge, UK), from February 2017 to August 2017. Male and female (nonchildbearing potential) subjects between the ages of 18 and 50 were eligible to be recruited, if they were deemed to be healthy by an experienced physician, had a body mass index within the range of $18-31 \mathrm{~kg} / \mathrm{m}^{2}$, and had blood chemistry, electrocardiogram, and spirometry results within normal range. Subjects were required to be current daily cigarette smokers and had to be able to produce $>100 \mathrm{mg}$ of sputum on saline induction at screening. Subjects were excluded if they had significant ongoing medical conditions, a recent history of asthma, or currently had asthma. Written informed consent was obtained prior to study participation. The study was conducted in accordance with the Declaration of Helsinki, and relevant ethics committee/institutional review boards and regulatory authorities reviewed and approved the study protocols.

Study Design. This study was a single-center, double-blind, placebo-controlled two-part study to evaluate the safety, tolerability,
PK, and PD of GSK2292767A in healthy participants who smoke cigarettes. Part A ( $n=16$ per cohort) used a two interlocking cohort, three treatment period crossover design to evaluate ascending single doses $(50,100,200,500,1000$, and $2000 \mu \mathrm{g})$ of GSK2292767A or placebo, with a 4 -week washout period between doses. Part B used a parallel group design ( $n=12$; 3:1 ratio; note: 11 subjects from part $\mathrm{A}$ participated in part B) to evaluate the highest tolerated dose from part A $(2000 \mu \mathrm{g})$ or placebo for 14 days of repeat dosing. In both parts, GSK2292767A was administered as a dry powder blended with lactose, and 1\% magnesium stearate using an ELLIPTA dry powder inhaler; placebo (lactose only) was administered using a matching device.

In part A, hypertonic saline-induced sputum samples were collected 3 and 24 hours after administration of inhaled treatment and blood samples for PK analysis were collected at predose, 5, 30, and 45 minutes, and 1, 2, 3, 4, 6, 8, 12, and 24 hours postdose. In part B, hypertonic saline-induced sputum samples were collected predose, 3 hours postdose on day 1 , and 3 and 24 hours postdose on day 12 of treatment. Blood samples for PK analysis were collected predose and 5 minutes postdose on days 2-13 inclusive, and predose, 5, 30, and 45 minutes, and 1, 2, 3, 4, 6, 8, and 12 hours postdose on days 1 and 14 . Bronchoscopy for the collection of BAL samples was performed on day 15 at 24 hours (trough) after the final (day 14) dose. Saline washes ( $3 \times$ $50 \mathrm{ml}$ ) were used for the lavage procedure via a bronchoscope that was positioned at the second bifurcation of the right middle lobe, and each return was recorded and kept separately on ice prior to processing.

\section{Study Procedures}

Blood Sample for PK. For the PK analysis of GSK2292767A, whole blood samples of approximately $3 \mathrm{ml}$ were collected for measurement of plasma concentrations of GSK2292767A. Blood samples were collected via venipuncture or a cannula into uniquely labeled $3 \mathrm{ml}$ K2EDTA collection tubes (Greiner) and placed in ice water, centrifuged, and refrigerated at approximately $1500 \mathrm{~g}$ for 10 minutes at $4^{\circ} \mathrm{C}$. Resultant plasma was transferred into Nunc tubes (ThermoFisher Scientific) and stored at $-20^{\circ} \mathrm{C}$ before shipment to the bioanalysis site.

BAL Sample for PK. BAL supernatant and cells were prepared by centrifuging the BAL at $750 \mathrm{~g}$ for 10 minutes. The supernatant was then removed (BAL supernatant), and the remaining pellet was reconstituted in deionized water as BAL cells. The volume of deionized water for reconstitution was equivalent to $20 \%$ of the recovered lavage volume following bronchoscopy. Prior to use, the reconstituted pellet was frozen at $-80^{\circ} \mathrm{C}$ to ensure complete lysing of the cells and stored at $-80^{\circ} \mathrm{C}$. The reconstituted BAL cell sample was defrosted thoroughly and mixed prior to analysis for total cell fraction concentration and intracellular drug level detection.

\section{Sample Analyses}

Drug Concentration Measurements in Plasma and BAL Cells and Fluid. Human plasma, BAL supernatant, and BAL cell pellet samples were analyzed for GSK2292767A using a validated analytical method based on protein precipitation, followed by highperformance liquid chromatography (LC-MS/MS) analysis. The lower limit of quantification was $20 \mathrm{pg} / \mathrm{ml}$ and the higher limit of quantification was $10,000 \mathrm{pg} / \mathrm{ml}$ when using a $50 \mu \mathrm{l}$ aliquot of human plasma, lung BAL supernatant, and BAL cell pellet.

Human plasma and lung BAL supernatant samples were analyzed for urea by a standard validated analytical method based on the RochRamel enzymatic reaction, using the urease and glutamate dehydrogenase methodology on the Siemens Advia 1800 chemistry analyzer. In plasma, the lower limit of quantification was $0.2 \mathrm{mmol} / \mathrm{l}$ with a higher limit of quantification of $239.5 \mathrm{mmol} / \mathrm{l}$, and in the $\mathrm{BAL}$ supernatant the lower limit of quantification was $0.01 \mathrm{mmol} / \mathrm{l}$ with a higher limit of quantification of $2.8 \mathrm{mmol} / \mathrm{l}$.

PK Analysis of Concentration Data. Individual plasma GSK2292767A concentration-time data using actual times were 
TABLE 1

Equilibrium solubility, permeability, and tissue binding data

\begin{tabular}{lcc}
\hline \multicolumn{1}{c}{ Parameter } & GSK2292767 & Nemiralisib \\
\hline Passive permeability across an MDCK monolayer at $\mathrm{pH} 7.4(\mathrm{~nm} / \mathrm{s})$ & 213 & 157 \\
SLF solubility $(\mu \mathrm{g} / \mathrm{ml})$ of micronized crystalline material at $4 \mathrm{~h}$ & 4 (free base), 27 (sodium salt), & 457 (hemisuccinate) \\
& 36 (cinnamate salt) & 97.9 \\
Rat lung tissue binding $(\%$ bound) & 96.3 & 99.2 \\
Mouse lung tissue binding (\% bound) & 94.6 & 97.8 \\
Mouse blood binding (\% bound) & 96.7 \\
\hline
\end{tabular}

MDCK, Madin-Darby canine kidney; SLF, simulated lung fluid.

analyzed using Phoenix WinNonlin (Certara Inc.) to derive PK parameters, including maximum observed plasma concentration $\left(C_{\max }\right)$, time to $C_{\max }\left(t_{\max }\right)$, trough concentration $\left(C_{\min }\right)$, and area under the plasma concentration-time curve $\left(\mathrm{AUC}_{(0-\mathrm{t})}\right)$. Assessment of dose proportionality was carried out for the plasma PK data using a power model. An estimate of the mean slope of $\log _{\mathrm{e}}(\mathrm{dose})$ was reported for $C_{\max }$ and $\mathrm{AUC}_{(0-\mathrm{t})}$, along with corresponding $90 \%$ confidence intervals (CIs). Further exploratory analysis was performed using the ANOVA method after normalizing the parameters by a chosen nominal dose of $100 \mu \mathrm{g}$. Adjusted geometric means and 90\% CIs were calculated for each dose along with estimated treatment ratios and corresponding $90 \%$ CIs.

Assessment of accumulation for the plasma PK data was carried out using data from the single (day 1) and repeat dose phases in part B. Accumulation ratios were calculated for both peak (geometric mean $C_{\text {max }}$ ) and trough (geometric mean $C_{\min }$ ) values using the day 14 and 1 values. Peak:trough ratios were also calculated for days 1 and 14 .

No formal assessment of steady-state was performed. Visual analysis of the concentration-time profiles taken daily during the studies was used to assess the achievement of steady-state concentration in the plasma of dosed subjects.

BAL samples (cells and supernatant) were analyzed for GSK2292767A concentrations for derivation of lung epithelial lining fluid (ELF) and cell pellet concentrations (part B only). Urea levels were measured in the BAL supernatant samples together with the corresponding timematched plasma sample for calculation of the ELF dilution factor (using urea as a dilution marker). The dilution factor was used to multiply the BAL GSK2292767A concentration to derive the volume of ELF within the sample, and subsequently concentrations within ELF. The derived ELF concentrations of GSK2292767A for each BAL wash were calculated separately. A pooled analysis was calculated per subject by calculating the total mass of GSK2292767A in all three washes and dividing by the total volume of ELF within all three washes. The cell pellet concentration (part B only) was derived using the resulting ELF concentration and the ratio of the raw (not urea corrected) sample results between supernatant and cell pellet (reconstituted and lysed).

Analysis of Intracellular Drug Detection. The intracellular drug assessment was based on the LiveCell mass spectrometry protocol described previously (Masujima, 2009). BAL samples for intracellular mass spectrometry analysis were stored at $4^{\circ} \mathrm{C}$ and assayed within 24 hours of collection. Briefly, $500 \mu \mathrm{l}$ was mixed with $1.5 \mathrm{ml}$ of PBS and plated on 35-mm-diameter, $0.17-\mathrm{mm}$ base thickness cell culture dishes and maintained at $37^{\circ} \mathrm{C}$ for 12 hours. The latter was for the cells to settle at the bottom of the dish, since sampling is dependent on cell adherence to the dish. Intracellular contents were captured using N-150 CT-1 Humanix tips and an Olympus X81 microscope coupled to a Scientifica Motorized Micromanipulator. Sampled tips were frozen at $-80^{\circ} \mathrm{C}$ and kept in dry ice until the mass spectrometry analysis was performed. Just before the analysis, $8 \mu \mathrm{l}$ of internal standard solution (20 nM fumaric acid and $20 \mathrm{nM}$ taurocholic acid in 50:50 methanol) was added to the back of the Humanix tip and the intracellular contents were analyzed using a Fusion Lumos Orbitrap mass spectrometer using a direct infusion setup. The analysis was performed in full scan-only mode, and the mass resolution of the Orbitrap was set to 250,000. Data were analyzed using Compound discoverer 2.1 using both the KEGG and Chemspider processing nodes.

Phosphatidylinositol-Biphosphate and PhosphatidylinositolTrisphosphate Detection in Induced Sputum Samples. Phosphatidylinositol-trisphosphate (PIP3) peak area proportion, i.e., PIP3 peak area/[PIP3 peak area + phosphatidylinositol-biphosphate (PIP2) peak area], was calculated from the mass spectrometer peak areas for PIP3 and PIP2, derived using established methodology (Clark et al., 2011). The PIP3 proportion, using PIP2, was calculated to correct for changes in cell numbers between different sputum samples, assuming PIP2 was correlated with the cell number (Clark, 2011).

Statistical Analysis of PIP3 Proportion. For study samples, $\log _{\mathrm{e}}$ (PIP3 peak area proportion) was analyzed using mixed effects, repeated measures analyses. Treatment ratios (and 90\% CI) of adjusted geometric means for GSK2292767A versus placebo are presented. Statistical models were used to determine Bayesian posterior probabilities (assuming a noninformative prior); a Student's $t$ test cumulative distribution function was used to obtain the probabilistic statements.

\section{Results}

Comparison of In Vitro Solubility and Permeability between GSK2292767A and Nemiralisib. GSK2292767 was demonstrated to have high permeability and low solubility, with the free base (GSK2292767A) displaying the lowest solubility of the salt forms investigated. Nemiralisib also displayed high permeability, but much greater solubility and very high lung tissue binding in the rat (Table 1). Individual dissolution rate profiles of each salt are shown in Supplemental Fig. 1.

Lung and Blood PK Following Pulmonary Administration of Aqueous Suspension and Dry Powder Material in Rodents. Following intranasal administration to the mouse, analysis of the aqueous dose confirmed nemiralisib to be completely in solution, whereas the lower solubility compound GSK2292767A was only $56 \%$ in solution in the $0.2 \%$ Tween 80/saline $\mathrm{pH} 4.5$ dose formulation. Both compounds rapidly appeared in blood with $t_{\max }$ occurring at the earliest time point sampled (10 minutes). Despite this, both compounds showed selectivity for lung tissue following deposition into the lung, with an apparent steady-state tissue partition achieved by 3 hours postdose; this was characterized by a high unbound lung-to-blood concentration ratio of $\sim 27$ (average across 5-8 hours) for nemiralisib and very high total lung-toblood concentration ratios of $\sim 3600$ and 40 for GSK2292767A and nemiralisib, respectively (Fig. 1). It was not possible to derive unbound lung concentrations for GSK2292767A because it was administered as a suspension rather than a solution.

When comparing the free base form of GSK2292767A with more soluble forms, i.e., sodium and cinnamate salts, enhanced lung retention was primarily observed after delivery of 
A

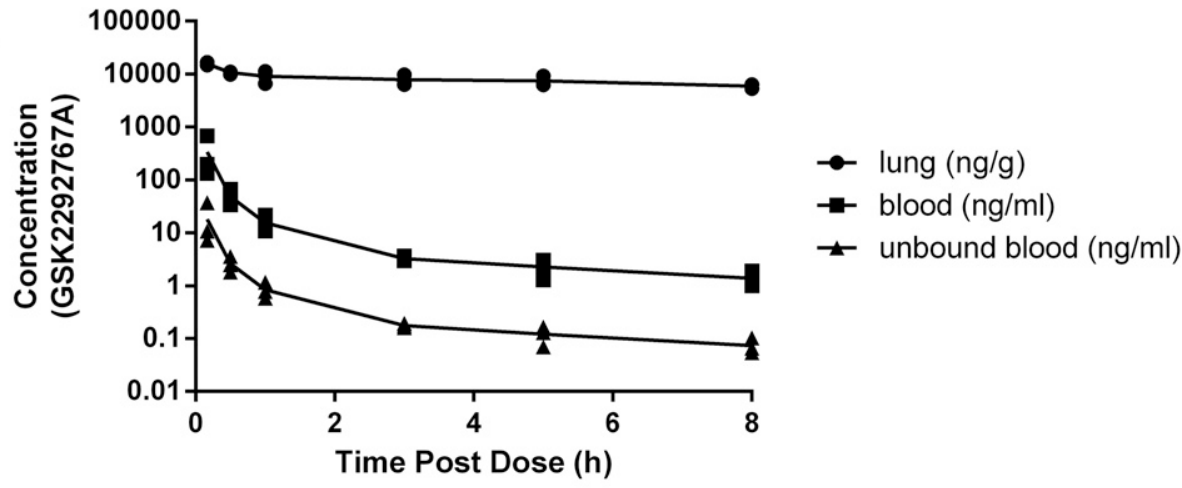

B

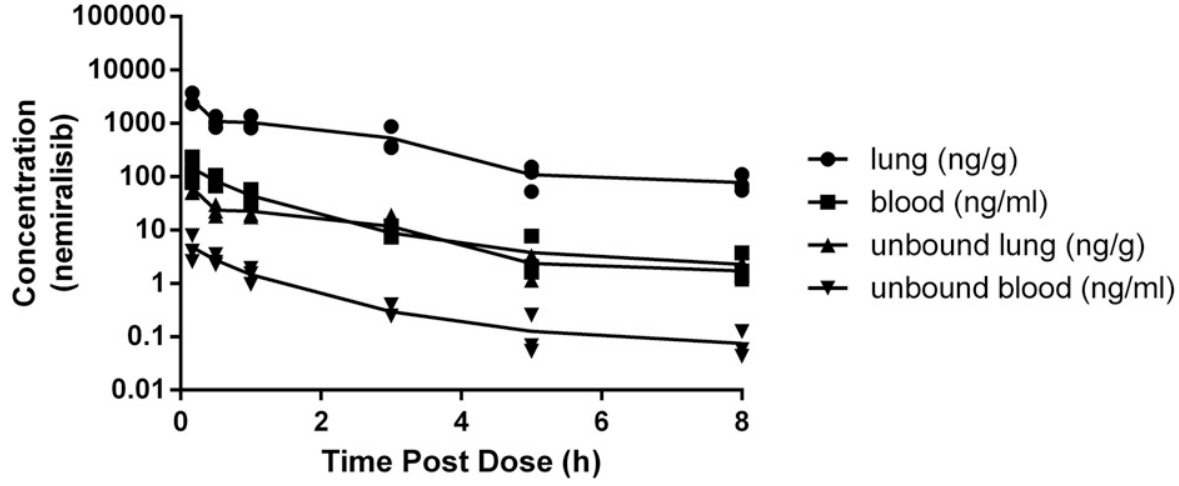

Fig. 1. Lung and blood PK following aqueous intranasal administration to the mouse. Lung disposition and systemic exposure of GSK2292767A (A) and nemiralisib (B) in the female BALB/c mouse following a single dose of $1 \mathrm{mg} / \mathrm{kg}$ administered intranasally. Profiles are displayed as total concentration and unbound concentration (with the exception of GSK2292767A lung for which unbound concentrations could not be estimated) derived by correcting for in vitro binding data generated in mouse whole blood and mouse lung homogenate. the free base GSK2292767A as an inhaled micronized lactose blend dry powder to rats. The lung retention of GSK2292767A was confirmed to correlate with reduced solubility (Fig. 2). An elimination half-life in the lung of approximately 9 hours was calculated for the selected free base; this, together with an increased plasma half-life following inhaled dry powder over intravenous administration (4.3 hours vs. 2.6 hours), indicated that the lung retention following pulmonary delivery is driven by slow dissolution in situ. The lung PK profile for nemiralisib following administration as a dry powder to the rat is included in Fig. 2 for comparison, highlighting that lung retention, although greater for GSK2292767A, was also achieved for nemiralisib. This is in keeping with the basicity and the high lung tissue binding displayed by nemiralisib.

GSK2292767A and Nemiralisib Inhibit Eosinophilia in a Murine Model of Pulmonary Inflammation. Both compounds were administered as inhaled dry powders in a HDM rechallenge model of pulmonary inflammation. Both GSK2292767A and nemiralisib displayed efficacy in this model when the rechallenge was given 12 hours after the last of seven daily doses, inhibiting BAL eosinophils collected 24 hours post rechallenge by $63 \%(P=0.015)$ and $74 \%(P=0.033)$, respectively (Fig. 3). Mean total lung drug levels for GSK2292767A measured on day 7 were $688 \mathrm{ng} / \mathrm{g}$ immediately after dose, $20 \mathrm{ng} / \mathrm{g} 18$ hours postdose ( 6 hours post rechallenge), and $8 \mathrm{ng} / \mathrm{g} 36$ hours postdose ( 24 hours post rechallenge). For nemiralisib, the levels were $630 \mathrm{ng} / \mathrm{g}$ immediately after dose, $10 \mathrm{ng} / \mathrm{g} 18$ hours postdose (6 hours post rechallenge), and below the level of detection 36 hours postdose (24 hours post rechallenge). Total lung levels determined immediately after dose were consistent on days 1 and 7 of dosing for both compounds.

\section{First Time in Human Study with GSK2292767A}

Demographics and Safety Data. In total, 37 subjects were randomized. All subjects were healthy volunteers who were current smokers (Table 2). The drug was generally well

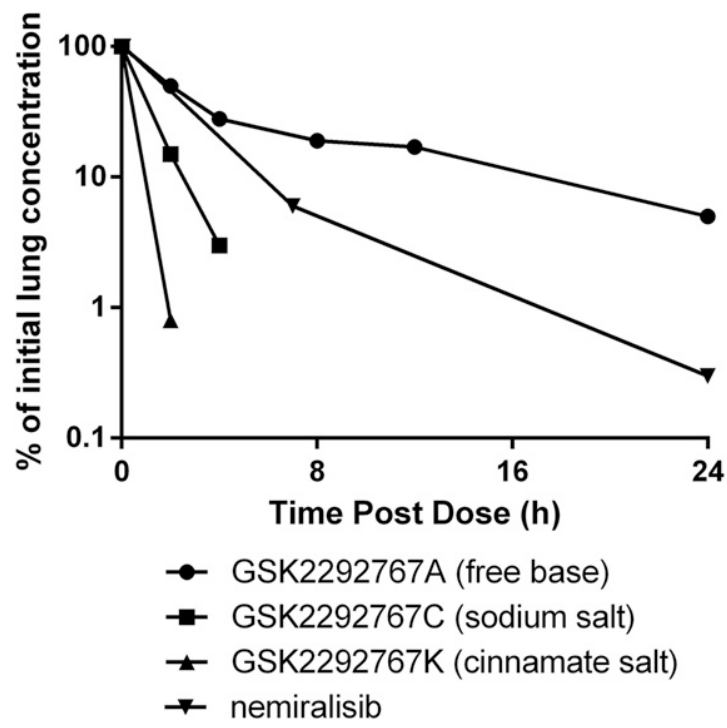

Fig. 2. Time course of percentage of total lung concentration measured immediately after dose following dry powder administration of different salt forms of GSK2292767 and nemiralisib (GSK2269557H) to the rat GSK2292767 salt forms delivered via an aerosolized dust generation inhalation tower with a Wright's dust feeder. Doses derived from filter analysis were $5,4.5$, and $1.5 \mathrm{mg} / \mathrm{kg}$ for the free base (GSK2292767A), sodium salt (GSK2292767C) and cinnamate salt (GSK2292767K), respectively. Nemiralisib (GSK2269557H, hemi-succinate salt form) data following dry powder delivery of $0.67 \mathrm{mg} / \mathrm{kg}$ via a Penn-Century device included for comparison. 


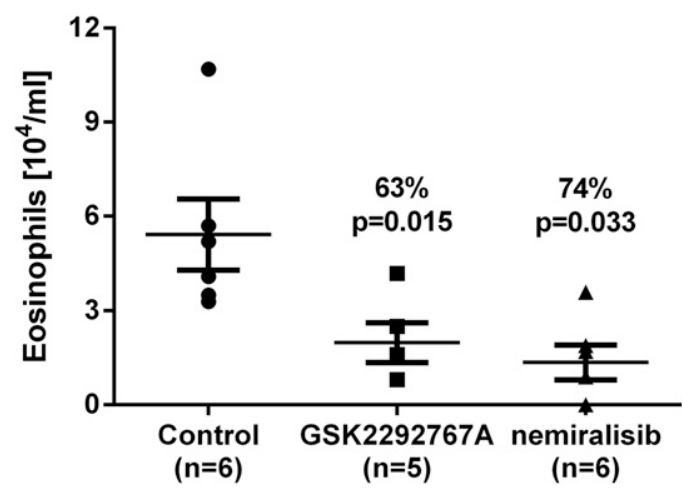

Fig. 3. GSK2292767A and nemiralisib inhibit eosinophilia in the HDM rechallenge model of pulmonary inflammation in the mouse. Inhibition of BAL eosinophil numbers 24 hours post HDM rechallenge, with HDM rechallenge administered 12 hours after the last of seven repeat once-daily doses of either lactose, GSK2292767A $(0.042 \mathrm{mg} / \mathrm{kg})$, or nemiralisib (GSK2269557H, $0.093 \mathrm{mg}$ base equivalent/kg) as a dry powder inhalation over 20 minutes on each occasion. Error bars represent S.E.M.; $P$ value derived using Dunnett's test.

tolerated and there were no incidences of any serious adverse events. All reported adverse events (AEs) were of mild-tomoderate severity and were resolved during the study (Table 3).

During the single ascending doses (part A) of the study, the no dose response was observed for the incidence of AEs and the number of subjects experiencing AEs were highest in the $50 \mu \mathrm{g}$ group (nine subjects, $75 \%$ ) compared with the other groups. The most commonly reported $\mathrm{AE}$ was headache, which was also reported by three subjects when receiving placebo. One subject from part A was reported to have an AE of idioventricular rhythm that led to subject withdrawal, but this was not considered to be related to the study drug.

The commonly reported AEs during the multiple daily dosing (part B) part of the study on $2000 \mu \mathrm{g}$ dose was contact dermatitis, followed by venipuncture site bruising. The $\mathrm{AE}$ of headache was observed in 12 subjects (part A) and 1 subject (part B), and was considered to be study drug related by the investigator during the blinded review of the data. Although there were some fluctuations observed around the normal ranges in the laboratory data, none of them were of any clinical significance or associated with any symptoms. No trends were observed for any of the clinical safety/laboratory parameters, vital signs, or ECG abnormalities.

During repeat dose of $2000 \mu \mathrm{g}$ once daily for 14 days, five incidental bronchoscopy findings of epithelial erythema were reported across both groups, with greater incidence for those on placebo. In general, the safety profile of GSK2292767A was similar to that previously observed with nemiralisib (Begg et al., 2019).

Single Dose PK (Plasma). Following single inhaled administration of doses of 50-2000 $\mu \mathrm{g}$ of GSK2292767A, the PK of GSK2292767A is characterized by an absorption phase reaching peak within approximately 1 hour (median $t_{\max }$ $0.75-1$ hours), followed by a biexponential decline in drug levels with geometric mean terminal phase elimination halflives ranging from 1.67 to 6.32 hours across the 50-2000 $\mu \mathrm{g}$ dose levels (Fig. 4). Exposures increased in an approximately proportional manner in both parts A and B with the geometric mean values presented in Table 4 .

TABLE 2

Subject disposition and demographics for parts A and B

\begin{tabular}{|c|c|c|c|c|}
\hline \multirow{2}{*}{ Parameter } & \multirow{2}{*}{$\begin{array}{c}\text { Part A } \\
\text { Total }\end{array}$} & \multicolumn{3}{|c|}{ Part B } \\
\hline & & Placebo & $2000 \mu \mathrm{g}$ & Total \\
\hline \multicolumn{5}{|l|}{ Number of subjects } \\
\hline Number of subjects planned $(N)$ & 32 & 3 & 9 & \\
\hline Number of subjects randomized $(N)$ & 37 & 3 & 9 & \\
\hline Number of subjects completed as planned $[n(\%)]$ & $29(78)$ & $3(100)$ & $9(100)$ & \\
\hline Number of subjects withdrawn (any reason) $[n(\%)]$ & $8(22)$ & $0(0)$ & $0(0)$ & \\
\hline Number of subjects withdrawn for $\mathrm{AE}[n(\%)]$ & $1(3)$ & & & \\
\hline \multicolumn{5}{|l|}{ Other reasons for subject withdrawal $[n(\%)]$} \\
\hline Withdrew consent & $2(5)$ & & & \\
\hline Investigator discretion & $5(14)$ & & & \\
\hline Positive drug screen & $2(5)$ & & & \\
\hline Withdrawn due to raised/elevated ALT & $2(5)$ & & & \\
\hline Chest tightness overnight & $1(3)$ & & & \\
\hline Demographics & $(n=37)$ & $(n=3)$ & $(n=9)$ & $(n=12)$ \\
\hline Age in years [mean (S.D.)] & $34.4(8.73)$ & $30.3(3.21)$ & $35.4(10.01)$ & $34.2(8.95)$ \\
\hline \multicolumn{5}{|l|}{$\operatorname{Sex}[n(\%)]$} \\
\hline Female & 0 & 0 & 0 & 0 \\
\hline Male & $37(100)$ & $3(100)$ & $9(100)$ & $12(100)$ \\
\hline BMI $\left(\mathrm{kg} / \mathrm{m}^{2}\right)[$ mean (S.D.)] & $25.92(3.22)$ & $22.94(2.81)$ & $26.05(3.40)$ & $25.27(3.43)$ \\
\hline Height $(\mathrm{cm})$ [mean (S.D.)] & $180.49(7.07)$ & $182.33(10.02)$ & $183.78(6.70)$ & $183.42(7.17)$ \\
\hline Weight (kg) [mean (S.D.)] & $84.44(11.73)$ & $76.27(10.83)$ & $88.11(13.51)$ & $85.15(13.52)$ \\
\hline \multicolumn{5}{|l|}{ Ethnicity $[n(\%)]$} \\
\hline Hispanic or Latino & $1(3)$ & 0 & $1(11)$ & $1(8)$ \\
\hline Not Hispanic or Latino & $36(97)$ & $3(100)$ & $8(89)$ & $11(92)$ \\
\hline \multicolumn{5}{|l|}{ Race $[n(\%)]$} \\
\hline African American/African Heritage & $2(5)$ & & & \\
\hline Asian: Central/South Asian Heritage & $1(3)$ & & & \\
\hline White: White/Caucasian/European Heritage & $34(92)$ & $3(100)$ & $9(100)$ & $12(100)$ \\
\hline Screening FEV FE $_{1}$ (L)ean (S.D.)] & $4.35(0.69)$ & $4.27(0.14)$ & $4.63(0.72)^{a}$ & $4.53(0.63)^{b}$ \\
\hline Predicted normal $\mathrm{FEV}_{1}[(\%)$, mean (S.D.)] & $97(8.22)$ & $91(8.72)$ & $100(11.15)^{a}$ & $97(10.97)^{b}$ \\
\hline
\end{tabular}


TABLE 3

Nonserious adverse events by preferred term for both part A (single dose) and part B (repeat dose)

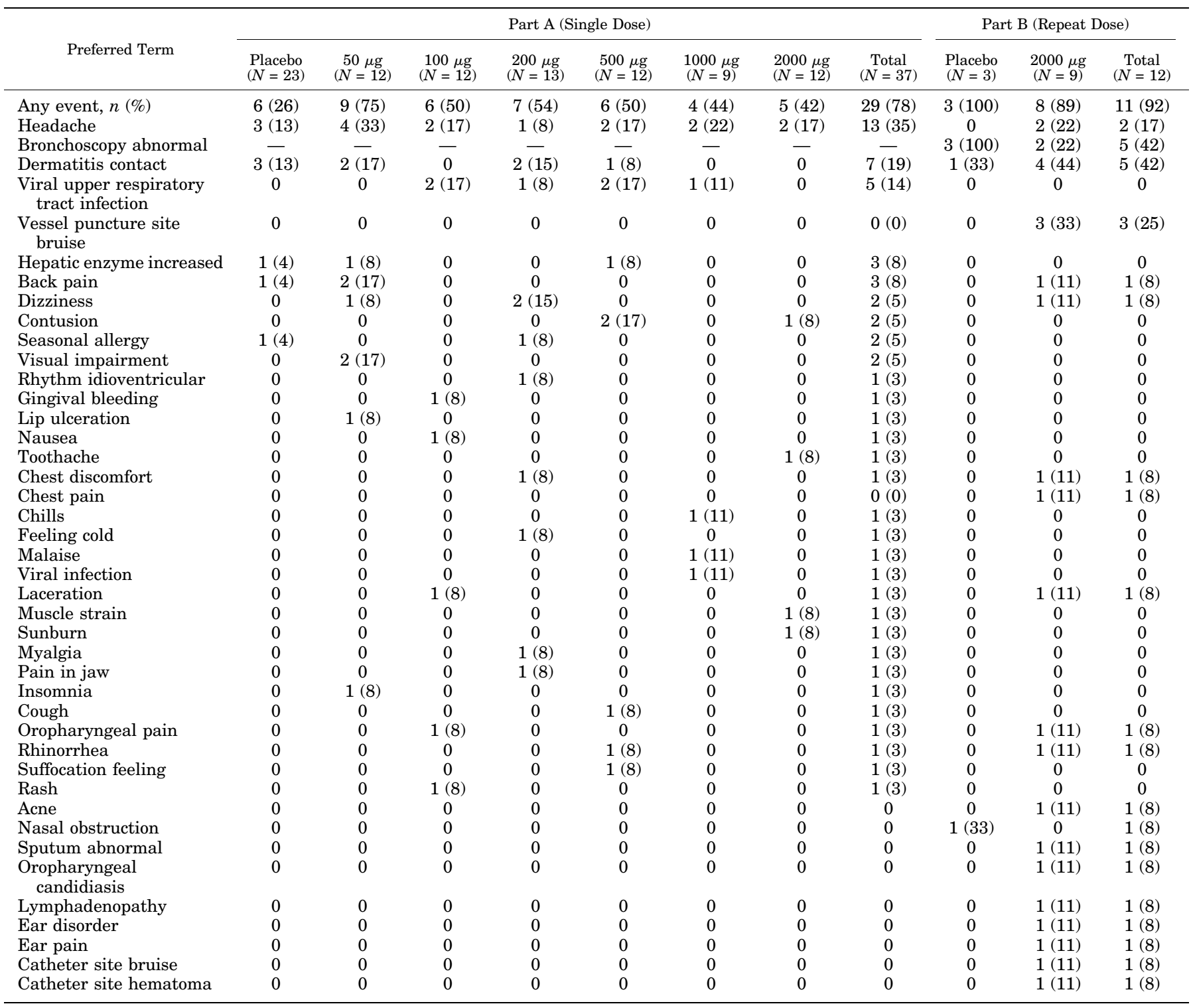

Bronchoscopy was not performed in Part A of the study.

Repeat Dose PK (Plasma). On repeat once-daily dosing at $2000 \mu \mathrm{g}$, GSK2292767A PK was predictable from single doses, with marginal accumulation in $C_{\max }$ and $\mathrm{AUC}_{(0-24)}$ values consistent with the calculated terminal phase elimination half-life after a single inhaled dose (Fig. 4). The $C_{\max }$ and AUC values showed moderate-to-high variability. The peakto-trough ratios (geometric mean $C_{\max }: C_{\min }$ ratios) were high for GSK2292767A, with ratios ranging from 16.15 (day 1) to 9.48 (day 14), but relatively constant between days 2 and 13 .

BAL PK (BAL Supernatant, Total Cell, and Intracellular Fractions). In part B following repeat daily dosing at $2000 \mu \mathrm{g}$, the concentrations of GSK2292767A in ELF values were derived from BAL supernatant data obtained 24 hours postdosing. Individual-derived ELF GSK2292767A concentrations were higher than the median plasma concentrations at the concurrent time point (24 hours). Arithmetic mean-derived ELF GSK2292767A concentrations were 133fold higher than GSK2292767A plasma concentrations at
24 hours postdose. Derived cell pellet concentrations were appreciably higher than derived ELF concentrations, as shown in Table 5. However, GSK2292767A was not detected in the intracellular fraction of BAL cells, whereas metabolites of nicotine (the subjects were smokers) and lignocaine (administered during bronchoscopy) were detected in the majority of samples (Supplemental Table 1).

GSK2292767A Reduces PIP3 Proportion in Induced Sputum. GSK2292767A reduced PIP3 proportions measured 3 hours after inhalation from adjusted geometric means of $0.00504(0.00467$ and 0.00544$)$ after receiving placebo to 0.00369 (0.00319 and 0.00426) following a single $2 \mathrm{mg}$ dose (Fig. 5). The ratio of adjusted geometric means for $2 \mathrm{mg}$ versus placebo was 0.73 (0.63 and 0.85), representing a 27\% decrease in the levels of PIP3 proportion with the probability that there truly is a decrease from placebo of $99.9 \%$. The reduced levels of PIP3 proportion were not maintained at 24 hours after receiving a single inhalation of the dose strengths administered. 

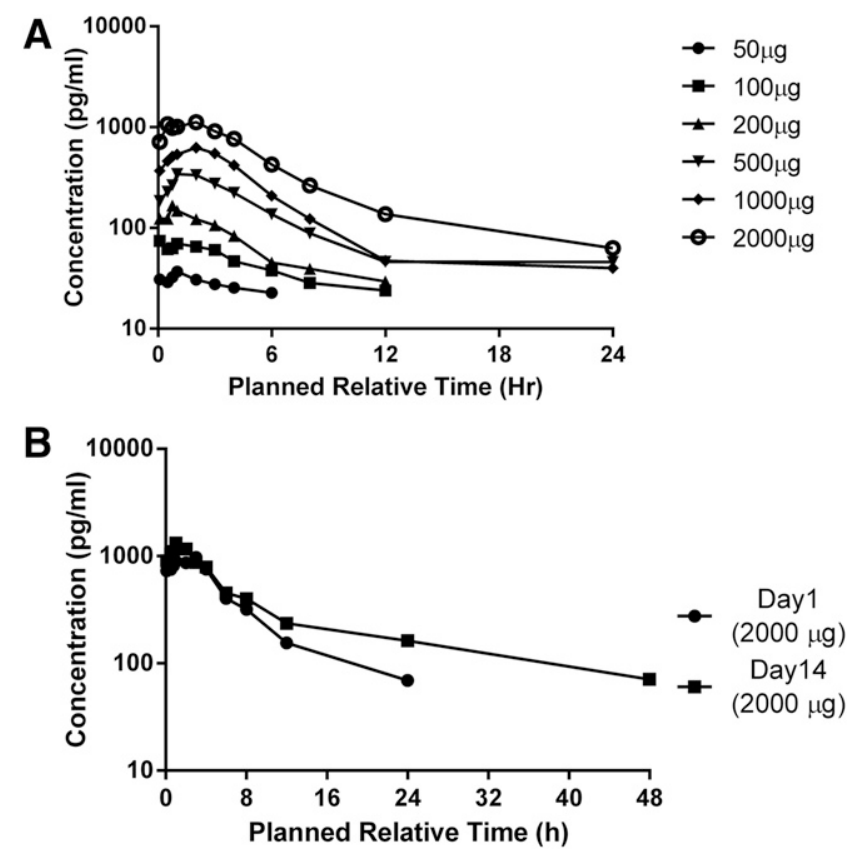

Fig. 4. Median plasma concentration-time plots after single and 14-day repeat inhaled dosing of GSK2292767A. Concentration-time plots following single inhaled dose administrations of 50-2000 $\mu \mathrm{g}$ GSK2292767A (A), and the comparison of single dose vs. 14-day repeat dosing of $2000 \mu \mathrm{g}$ GSK2292767A (B)

After 12 days of repeat dosing of $2 \mathrm{mg}$ of GSK2292767A, the PIP3 proportion reduced from the unadjusted geometric mean of $0.00500(0.00428$ and 0.00584$)$ at baseline to 0.00350 (0.00303 and 0.00405) 3 hours after the final dose, representing a $34 \%$ reduction with the probability that there truly is a decrease of $>99.9 \%$ (Table 6 ). This reduction in PIP3 was not maintained in the sputum sample obtained 24 hours after the 12-day dosing.

GSK2292767A Has a Direct Relationship between Exposure and PD Effect. From part B of the study, a scatter plot of individual subject's PIP3 proportion in sputum against respective time-matched GSK2292767A plasma concentration after single and repeat daily $2 \mathrm{mg}$ dosing is provided in Fig. 6. This shows a direct relationship between exposure and levels of PIP3.

\section{Discussion}

It is critical for drugs targeting the lung to be retained in the airways after inhalation to maintain target exposure. We have used two different drug optimization strategies to drive lung retention and present a detailed comparison of a novel inhaled inhibitor of PI3K $\delta$ (GSK2292767A) with the previously reported, structurally distinct $\mathrm{PI} 3 \mathrm{~K} \delta$ inhibitor nemiralisib. Evaluation in a first time in human study demonstrated that GSK2292767A was safe and well tolerated. The key finding of our detailed comparison of the in vitro, in vivo, and clinical profiles of GSK2292767A and nemiralisib was that our lung retention strategies were successful for both compounds; however, unexpectedly for GSK2292767A this did not translate into a prolonged target engagement profile. This has important implications for choice of lung retention strategies when optimizing drugs for inhaled delivery.

While lowering solubility is an effective strategy to drive lung retention for extended duration of inhaled molecules, our data raise the question of whether this is a desired approach, particularly where the drug target is intracellular. Analyses of compound in BAL and intracellular clinical samples, combined with a target engagement marker, were key factors in understanding the reason behind the lack of translation of the increased lung retention profile into prolonged target engagement with GSK2292767A. It was our observation that the total BAL cell drug concentration represented undissolved particulate material, drug bound to the outside of cells, and drug free to engage with the target. To determine the relevant drug concentration, one would need to determine the intracellular drug concentration available to interact with the target. Therefore, measuring this in a clinical trial in combination with matching preclinical approaches is recommended to fully understand the profile of any novel inhaled molecule.

TABLE 4

Summary of plasma PK from parts A and B following single and repeat dosing with GSK2292767A

\begin{tabular}{cccccc}
\hline PK Parameter & Treatment & $N$ & $n$ & Geometric Mean & $95 \%$ CI \\
\hline$U$ & $\mu g$ & & & & \\
Part A (single dose) & & & & & \\
AUC $_{(0-24)}\left(\mathrm{h}^{*}\right.$ pg/ml) & 50 & 12 & 0 & $\mathrm{NE}$ & $\mathrm{NE}$ \\
& 100 & 12 & 2 & 596 & $(242,1467)$ \\
& 200 & 13 & 5 & 722 & $(370,1408)$ \\
& 500 & 12 & 10 & 2460 & $(1564,3871)$ \\
& 1000 & 9 & 8 & 3690 & $(2756,4940)$ \\
& 2000 & 12 & 11 & 7781 & $(5587,10,838)$ \\
$C_{\max }(\mathrm{pg} / \mathrm{ml})$ & 50 & 12 & 12 & 36.9 & $(30.3,45.0)$ \\
& 100 & 12 & 12 & 90.9 & $(75.2,110)$ \\
& 200 & 13 & 12 & 171 & $(131,223)$ \\
& 500 & 12 & 12 & 356 & $(430,453)$ \\
& 1000 & 9 & 9 & 580 & $(1009,1741)$ \\
Part B $\left(\mathrm{repeat}\right.$ dosing) $_{\text {AUC }}$ & 2000 & 12 & 12 & 1325 & \\
$C_{(0-24)}(\mathrm{h}$ pg/ml) & & & & & $(4764,13,989)$ \\
& 2000 & Day 1 & 8 & 8163 & $(819,1603)$ \\
& 2000 & Day 14 & 9 & 9942 & $(958,1779)$
\end{tabular}

$C_{\max }$, maximum observed plasma concentration; $N$, total number of subjects; $n$, total number of subjects with sufficien samples with detectable levels of drug; $\mathrm{NE}$, not evaluable. 
TABLE 5

Summary of derived lung epithelial lining fluid and cell pellet GSK2292767A pharmacokinetic concentrations

\begin{tabular}{lcccll}
\hline \multicolumn{1}{c}{ Measurement } & $N$ & $n$ & Geometric Mean & 90\% CI & Fold Change vs. Nemiralisib $^{a}$ \\
\hline & & & $n g / m l$ & & \\
Day 14 plasma $C_{\text {min }}$ & 9 & 9 & 0.06 & $(0.03,0.09)$ & Decrease $(\sim 29$-fold $)$ \\
Pooled epithelial lining fluid drug concentration & 9 & 8 & 8.85 & $(3.53,22.22)$ & Decrease $(\sim 6$-fold) \\
Derived cell pellet concentration & 9 & 8 & 17,572 & $(8278,37,303)$ & Increase $(\sim 48$-fold $)$ \\
\hline
\end{tabular}

$C_{\min }$, trough concentration; $N$, total number of subjects; $n$, total number of subjects with sufficient samples with detectable levels of drug. ${ }^{a}$ Compared with previously reported data (Begg et al., 2019).

However, understanding the PK/PD relationship for lowsolubility inhaled compounds is complex. The total lung and blood concentration profiles shown in Fig. 1 demonstrate clear selectivity for lung tissue with both compounds in a preclinical setting. Importantly, lung selectivity could be confirmed for nemiralisib through derived unbound concentrations because nemiralisib was dosed as a solution. Nemiralisib displayed an unbound lung:blood ratio of 27; calculated across the latter part of the time concentration profile. However, it is more difficult to derive unbound lung concentrations for GSK2292767A following pulmonary administration as a suspension. With around one-half of the delivered GSK2292767A dose being undissolved, total lung concentrations include particulate material unavailable for binding to lung tissue or indeed the intracellular therapeutic target. Consequently, any attempt to derive
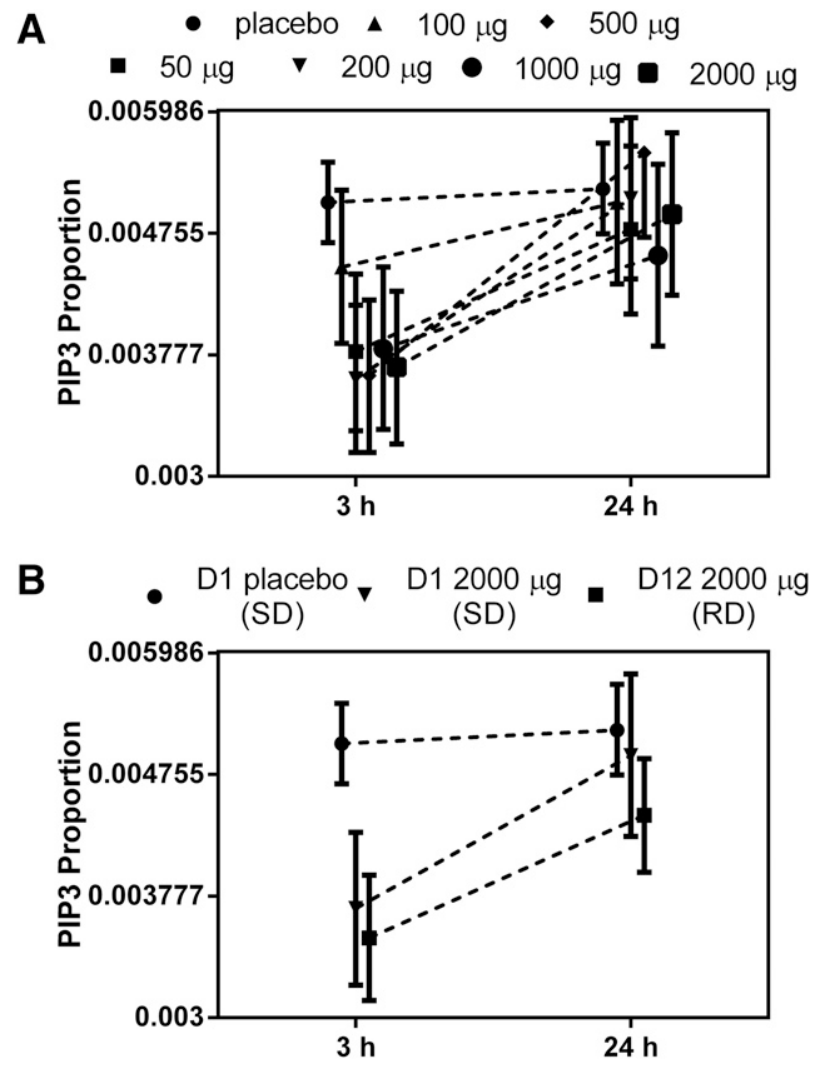

Fig. 5. GSK2292767A reduced PIP3 levels in induced sputum 3 and 24 hours post single and repeat dose GSK2292767A reduced the PIP3 levels in induced sputum samples in healthy smokers who were given single dose administration (A) and 12-day repeat dose administration (B). $\mathrm{SD}=$ single dose and $\mathrm{RD}=$ repeat dose at 3 hours post dose, but not 24 hours post dose. unbound lung concentrations for GSK2292767A would be an overestimate (Cooper et al., 2012).

The free base form of GSK2292767A was selected because it had longer lung retention compared with the more soluble sodium and cinnamate salt forms (see Fig. 2). The risk of accumulation often associated with poorly soluble compounds upon repeat dosing (Owen, 2013; Lewis et al. 2014) was mitigated through a combination of high permeability and an appropriate dissolution profile, and proved successful. The data in Supplemental Fig. 1 show the low-solubility free base dissolving at a slower rate than the sodium and cinnamate salt forms. However, the constant removal of dissolved material under sink conditions allows the free base to dissolve throughout the duration of the dissolution test. This is in keeping with conditions in the lung, where the high permeability of GSK2292767A is expected to drive the rapid absorption of the dissolved fraction across the lungs. Assuming a similar dissolution profile in situ, this also supports the hypothesis that GSK2292767A offers slow release to the site of action in the lungs.

Based on the longer lung retention of the free base version, GSK2292767A was profiled in a murine pulmonary inflammation model. Using once-daily dosing, both GSK2292767A and nemiralisib demonstrated efficacy following HDM rechallenge, suggesting that both lung retention strategies successfully translated into sufficient target engagement to drive duration of effect in the mouse. Overall, the preclinical profile for GSK2292767A supported progression into a first time in human clinical trial to explore its safety, PK, and PD profiles as an alternative molecule to nemiralisib. Since nemiralisib had already been confirmed as a once-daily inhaled molecule based on target engagement, GSK2292767A provided the unique opportunity to compare the two compounds and their differentiated lung retention strategies in a clinical setting.

The drug was generally well tolerated, with headache being the only drug-attributed adverse effect observed during the single dose escalation phase. During the repeat dosing phase there were five incidental bronchoscopy findings of bronchial epithelial erythema reported across both active and placebo groups, which was most likely related to active smoking. Smoking is described to directly activate the PI3K $\delta$ pathway (To et al., 2010; Daijo et al., 2016).

Following single inhaled administration, GSK2292767A was rapidly absorbed with plasma levels declining after achieving $t_{\max }$ approximately 1 hour after dosing. Plasma $C_{\text {max }}$ and AUC were linear, increasing in an approximate doseproportional manner. Consistent with the estimated half-life, there was marginal 2-fold accumulation at trough on day 14 relative to day 1 . Consistent with preclinical observations, high levels of GSK2292767A were observed in the BAL cell 
TABLE 6

PIP3 levels in induced sputum following single and repeat dosing

\begin{tabular}{|c|c|c|c|c|}
\hline Dose & Time Point & Ratio of Adjusted Geometric Mean (90\% CI) vs. Placebo & Reduction in PIP3 proportion & Probability $($ ratio $<1)$ \\
\hline$\mu g$ & $h$ & & $\%$ & $\%$ \\
\hline \multicolumn{5}{|l|}{ Part A } \\
\hline \multirow[t]{2}{*}{50} & 3 & $0.75(0.64,0.88)$ & 25 & 99.8 \\
\hline & 24 & $0.93(0.78,1.10)$ & 7 & 76.8 \\
\hline \multirow[t]{2}{*}{100} & 3 & $0.88(0.76,1.03)$ & 12 & 90.5 \\
\hline & 24 & $0.97(0.82,1.15)$ & 3 & 59.8 \\
\hline \multirow[t]{2}{*}{200} & 3 & $0.71(0.61,0.84)$ & 29 & 100 \\
\hline & 24 & $0.98(0.83,1.17)$ & 2 & 56.7 \\
\hline \multirow[t]{2}{*}{500} & 3 & $0.72(0.61,0.84)$ & 28 & 100 \\
\hline & 24 & $1.07(0.90,1.28)$ & -7 & 26.1 \\
\hline \multirow{2}{*}{1000} & 3 & $0.76(0.64,0.89)$ & 24 & 99.7 \\
\hline & 24 & $0.88(0.73,1.06)$ & 12 & 86.7 \\
\hline \multirow[t]{2}{*}{2000} & 3 & $0.73(0.63,0.85)$ & 27 & 99.9 \\
\hline & 24 & $0.95(0.81,1.13)$ & 5 & 68.1 \\
\hline \multicolumn{5}{|l|}{ Part B } \\
\hline & 3 (day 1$)$ & $0.62(0.44,0.89)$ & $38^{a}$ & 98.3 \\
\hline \multirow{2}{*}{2000} & 3 (day 12) & $0.80(0.67,0.97)$ & $20^{a}$ & 96.9 \\
\hline & 24 (day 12 ) & $1.03(0.77,1.38)$ & $-3^{a}$ & 42 \\
\hline
\end{tabular}

${ }^{a}$ Placebo corrected values.

pellet fraction 24 hours post inhalation, more so than observed previously with nemiralisib. This would lead us to believe there were sufficient levels of GSK2292767A in the lung 24 hours after dosing to adequately inhibit PI3K $\delta$, based on previous data generated with nemiralisib.

However, to our surprise the robust target engagement detected shortly after dosing was not maintained. Induced sputum samples captured 3 hours after single inhaled dosing showed a reduction in PIP3, demonstrating that GSK2292767A had successfully been delivered into the lung and inhibited the kinase. However, these reductions were not meaningfully maintained at 24 hours, suggesting a single dose did not adequately engage the target for 24 hours. Reductions in PIP3 proportion were also observed to a similar degree upon repeat dosing, and despite drug being present in BAL the reduction was not observed at 24 hours. This suggested that the levels of GSK2292767A detected in the BAL cell pellet at 24 hours were either undissolved or bound to the outside of cells. This profile differs significantly from that of nemiralisib, which showed similar magnitude reductions in PIP3 proportion at both 3 and 24 hours after single and repeat dosing.

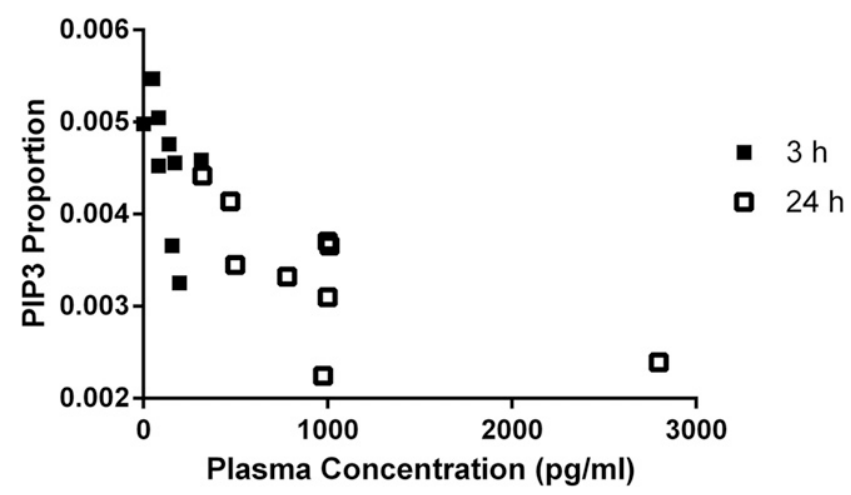

Fig. 6. GSK2292767A shows a linear relationship between exposure and pharmacodynamic effect. A scatter plot of plasma GSK2292767A concentration vs. PIP3 proportion 3 hours following single (closed squares) and 24 hours following 12-day repeated (open squares) inhaled dosing of $2000 \mu \mathrm{g}$ GSK2292767A suggest a direct relationship between the increase in plasma exposure of drug and the decrease in proportion of PIP3 levels.
We noted that levels in the BAL fluid fraction were lower for GSK2292767A compared with nemiralisib, indicating that drug levels at the target may not have been as high as suggested by the BAL cell pellet data. Analytically, the technique for measuring GSK2292767A in the BAL cell pellet is unable to differentiate between undissolved particulate material and the free versus bound drug. To directly address this, we extracted the intracellular contents of cells taken from the BAL and analyzed them (analyst blinded) for presence of GSK2292767A. Despite high levels of drug detected in the BAL cell pellet, GSK2292767A was undetectable inside BAL cells. This methodology is time consuming; hence, a limited number of individual cells were analyzed. However, metabolites of nicotine (the subjects were smokers) and lignocaine (applied topically to lung during bronchoscopy procedure) were detected, consistent with the population recruited into this study and increasing confidence in the methodology. The BAL fluid concentrations, being lower for GSK2292767A than for nemiralisib, are potentially a better surrogate measure for free concentration in the lung available to engage the drug target. It should be noted that BAL fluid concentrations could remain an overestimation of free drug concentration, for example, if particulate material is dissolved during the BAL sample capture or if significant protein binding is present.

In summary, this approach highlights the importance of generating a detailed analysis of exposure and response in the correct compartment to adequately interpret the human PK/PD relationship. Therefore, we recommend measuring drug levels in both fluid, total cell, and intracellular compartments to obtain a complete understanding of target exposure. This approach should also be employed in preclinical PK and PD models to generate translational data sets. This study has demonstrated that prolonged lung retention can indeed be achieved by lowering molecule solubility; however, if the drug target is intracellular, adopting this strategy may not result in sufficient target engagement to deliver a PD response. This study was a unique opportunity to compare and contrast two similar molecules with differing solubilities in matching clinical studies to directly explore the translation of inhaled drug optimization strategies. The outcomes should be used to inform future inhaled drug discovery approaches. 


\section{Acknowledgments}

The authors gratefully acknowledge editorial support in the form of language editing and manuscript formatting provided by Fiona Woodward, CMPP of Fishawack Indica Ltd., which was funded by GlaxoSmithKline. The authors acknowledge the contributions of Dr. Jurgen Herre for conducting bronchoscopies during the study at the Clinical Unit Cambridge, Andrew Want for assistance in biomarker sample handling, and the Clinical Unit Cambridge staff for recruiting subjects and setting up the bronchoscopy and sputum induction.

\section{Authorship Contributions}

Participated in research design: Begg, Edwards, Hamblin, Pefani, Wilson, Gilbert, Vitulli, Mallett, Morrell, Hingle, Uddin, Harrell, Newman, Fernando, Hessel.

Conducted experiments: Mallett, Morrell, Hingle, Uddin, Newman, Fernando, Clark.

Performed data analysis: Edwards, Pefani, Wilson, Gilbert, Vitulli, Mallett, Morrell, Hingle, Uddin, Harrell, Newman.

Wrote or contributed to the writing of the manuscript: Begg, Edwards, Hamblin, Pefani, Wilson, Gilbert, Vitulli, Mallett, Morrell, Hingle, Uddin, Ehtesham, Marotti, Harrell, Newman, Fernando, Clark, Cahn, Hessel.

\section{References}

Begg M, Wilson R, Hamblin JN, Montembault M, Green J, Deans A, Amour A, Worsley S, Fantom K, Cui Y, et al. (2019) Relationship between pharmacokinetics and pharmacodynamic responses in healthy smokers informs a once daily dosing regimen for nemiralisib. J Pharmacol Exp Ther, in press. https://doi.org/10.1124/jpet.118.255109.

Cahn A, Hamblin JN, Begg M, Wilson R, Dunsire L, Sriskantharajah S, Montembault M, Leemereise CN, Galinanes-Garcia L, Watz H, et al. (2017) Safety, pharmacokinetics and dose-response characteristics of GSK2269557, an inhaled PI3K $\delta$ inhibitor under development for the treatment of COPD. Pulm Pharmacol Ther 46:69-77.

Clark J, Anderson KE, Juvin V, Smith TS, Karpe F, Wakelam MJO, Stephens LR, and Hawkins PT (2011) Quantification of PtdInsP3 molecular species in cells and tissues by mass spectrometry. Nat Methods 8:267-272.

Cooper AE, Ferguson D, and Grime K (2012) Optimisation of DMPK by the inhaled route: challenges and approaches. Curr Drug Metab 13:457-473.

Daijo H, Hoshino Y, Kai S, Suzuki K, Nishi K, Matsuo Y, Harada H, and Hirota K (2016) Cigarette smoke reversibly activates hypoxia-inducible factor 1 in a reactive oxygen species-dependent manner. Sci Rep 6:34424.

Davies NM and Feddah MR (2003) A novel method for assessing dissolution of aerosol inhaler products. Int J Pharm 255:175-187.

Down K, Amour A, Baldwin IR, Cooper AW, Deakin AM, Felton LM, Guntrip SB Hardy C, Harrison ZA, Jones KL, et al. (2015) Optimization of novel indazoles as highly potent and selective inhibitors of phosphoinositide 3-kinase $\delta$ for the treatment of respiratory disease. $J$ Med Chem 58:7381-7399.

Glossop PA, Lane CA, Price DA, Bunnage ME, Lewthwaite RA, James K, Brown AD Yeadon M, Perros-Huguet C, Trevethick MA, et al. (2010) Inhalation by design: novel ultra-long-acting $\beta_{2}$-adrenoreceptor agonists for inhaled once-daily treatment of asthma and chronic obstructive pulmonary disease that utilize a sulfonamide agonist headgroup. J Med Chem 53:6640-6652.

Hochhaus G, Gonzalez-Rothi RJ, Lukyanov A, Derendorf H, Schreier H, and Dalla Costa T (1995) Assessment of glucocorticoid lung targeting by ex-vivo receptor binding studies in rats. Pharm Res 12:134-137.

Hochhaus G, Möllmann H, Derendorf H, and Gonzalez-Rothi RJ (1997) Pharmacokinetic/pharmacodynamic aspects of aerosol therapy using glucocorticoids as a model. J Clin Pharmacol 37:881-892.

Kalvass JC and Maurer TS (2002) Influence of nonspecific brain and plasma binding on CNS exposure: implications for rational drug discovery. Biopharm Drug Dispos 23:327-338.

Khindri S, Cahn A, Begg M, Montembault M, Leemereise C, Cui Y, Hogg A, Wajdner H, Yang S, Robertson J, et al. (2018) A multicentre, randomized, double-blind, placebo-controlled, crossover study to investigate the efficacy, safety, tolerability, and pharmacokinetics of repeat doses of inhaled nemiralisib in adults with persistent, uncontrolled asthma. J Pharmacol Exp Ther 367:405-413.

Lewis DJ, Williams TC, and Beck SL (2014) Foamy macrophage responses in the rat lung following exposure to inhaled pharmaceuticals: a simple, pragmatic approach for inhaled drug development. J Appl Toxicol 34:319-331.

Lipworth BJ (1996) Pharmacokinetics of inhaled drugs. Br J Clin Pharmacol 42 697-705.

Lötvall J (1997) Local versus systemic effects of inhaled drugs. Respir Med $\mathbf{9 1}$ (Suppl A):29-31.

Masujima T (2009) Live single-cell mass spectrometry. Anal Sci 25:953-960.

Owen K (2013) Regulatory toxicology considerations for the development of inhaled pharmaceuticals. Drug Chem Toxicol 36:109-118.

Perry MWD, Björhall K, Bonn B, Carlsson J, Chen Y, Eriksson A, Fredlund L, Hao H, Holden NS, Karabelas K, et al. (2017) Design and synthesis of soluble and cellpermeable PI3K $\delta$ inhibitors for long-acting inhaled administration. $J$ Med Chem 60:5057-5071.

Riley T, Christopher D, Arp J, Casazza A, Colombani A, Cooper A, Dey M, Maas J, Mitchell J, Reiners M, et al. (2012) Challenges with developing in vitro dissolution tests for orally inhaled products (OIPs). AAPS PharmSciTech 13:978-989.

Stark AK, Sriskantharajah S, Hessel EM, and Okkenhaug K (2015) PI3K inhibitors in inflammation, autoimmunity and cancer. Curr Opin Pharmacol 23: 82-91.

Strong P, Ito K, Murray J, and Rapeport G (2018) Current approaches to the discovery of novel inhaled medicines. Drug Discov Today 23:1705-1717.

To Y, Ito K, Kizawa Y, Failla M, Ito M, Kusama T, Elliott WM, Hogg JC, Adcock IM, and Barnes PJ (2010) Targeting phosphoinositide-3-kinase- $\delta$ with theophylline reverses corticosteroid insensitivity in chronic obstructive pulmonary disease. Am $J$ Respir Crit Care Med 182:897-904.

Address correspondence to: Dr. Edith M. Hessel, Refractory Respiratory Inflammation Discovery Performance Unit, GlaxoSmithKline, Stevenage, UK. E-mail: edith.m.hessel@gsk.com 